\title{
Design of Health Telemonitoring System on Vital Sign Patient's Web-Based
}

\author{
Mery Subito', Alamsyah' ${ }^{2}$, Ardi Amir ${ }^{3}$ \\ \{ subitomery@yahoo.com ${ }^{1}$, alamsyah.zakaria@untad.ac.id ${ }^{2}$, ardi.amir@yahoo.com ${ }^{3}$ \} \\ ${ }^{1,2,3}$ Department of Electrical Engineering, Tadulako University, Palu, Indonesia
}

\begin{abstract}
Wireless-based health monitoring systems are now becoming interesting to develop, especially monitoring vital signs such as respiration, heartbeat, brain signal, body temperature, and blood pressure. Vital sign monitoring is essential for doctors and nurses as a form of early prevention in maintaining patient safety, especially for elderly patients. Examination of heart rate and body temperature requires fast service and handling. However, the problem that occurs at this time is the time needed by doctors or nurses to provide diagnostic results long enough, the equipment used by nurses in examining patients generally still use the manual system (see and record patient conditions), and the number of medical personnel which limited with increasing numbers patients certainly slow down health services. To overcome these problems, the researcher proposes a device that is capable of monitoring vital sign patients in real time. The aim of this research is to help doctors and nurses improve patient health services, especially in showing the results of the heartbeat, minimizing the occurrence of diagnostic errors, and reducing the risk of death. The proposed design has an average accuracy in detecting heartbeat reach is $97.90 \%$ and blood pressure reach is $97.69 \%$.
\end{abstract}

Keywords: Health monitoring, sensor, blood pressure, body tempretaure, raspberry pi.

\section{Introduction}

The monitoring system is currently increasing and is interesting to study, especially in wireless-based monitoring systems. The application of the wireless-based monitoring system has been carried out in areas affected by natural disasters (forest fires, floods, and earthquakes), health (vital signs: monitoring heart rate, body temperature, and blood pressure), and health structures of bridges. The patient's vital sign examination is critical to be carried out as control of the physical condition information and the initial diagnosis of the disease suffered. Vital sign, especially heart disease based on data from the world health organization (WHO) ranks highest reaching $31 \%$ or 1.7 million people each year the cause of death globally caused by heart attacks. It shows a severe threat to world public health [1]. In 2014 in Southeast Asia, especially Indonesia the mortality rate reached 35\% due to heart disease. One of the factors that cause high mortality in heart disease is the slow handling of examination of the patient's disease condition.

The issue of vital signs indeed requires special attention for the government in this case medical personnel to reduce the death rate caused by abnormal body temperature, blood pressure, and heart rate. This parameter is an indicator that has the potential to cause heart attacks and patient deaths. Currently, health services in hospitals and community health centres related to vital sign equipment are available such as stethoscopes and vital signs monitors. However, the stethoscope has a lack of functional, and vital sign monitors that are still cable-based. This service system indeed requires time for medical personnel to check the 
condition of patients with increasing numbers of patients. This condition is not sufficient to be applied to health services with the number of patients always growing and the limited number of medical personnel who will control patients.

Based on the problems mentioned above, the researcher proposed a web-based patient's vital sign monitoring system. This paper aims to help medical personnel monitor patients' vital sign conditions, reduce the occurrence of diagnostic errors, optimally improve health services, especially the administration of health data, and reduce operational costs [2].

Several researchers have proposed research related to vital sign systems, including ZigBee-based heart rate monitoring [3], Android [4], radio frequency identification (RFID) [5], portable intelligent ECG monitor [6], and dry-contact electrodes [7]. However, the author only focuses on monitoring ECG not yet developing to blood pressure. Body-based ECG and temperature monitoring system based on Butterworth filter [8], android mobile application [9], Bluetooth [10], raspberry pi [11], however, the authors have not developed a blood pressure detection system. Likewise with the ECG monitoring system, body temperature, and blood pressure based on Bluetooth [12] which has not designed into a web-based system and the distance of data delivery range is only a maximum of 10 meters.

Research related to simulations has also proposed by several researchers including analysis of quality of service (QoS) on the AODV routing protocol, DSDV using NS2 [13] and OLSR using network simulator 3 (NS3). Simulation results show that OLSR is very suitable to be applied to a more substantial number of patients and AODV is very supportive of being referred to the environmental conditions of the human body. However, the researcher did not explain the devices used in the simulation parameters.

\section{Research Methods}

Monitoring systems such as blood pressure, heart rate, and body temperature are made to facilitate medical personnel in controlling and analysing the patient's condition. The stages of research carried out consisted of literature studies, system design (hardware and software), component manufacturing and installation, system testing, data retrieval, and system analysis. The research stages show in Figure 1.

\subsection{Study literature}

At this stage, a research reference carried out related to the vital sign and material used. Reference collection aims to support research, such as measurement of heart rate, body temperature, and blood pressure, standard measures of vital sign based on age and sex.

\subsection{System design}

The vital sign monitoring system starts with taking vital sign sensor data through the raspberry pi device. Sensor data will be processed and stored on the raspberry pi module. Furthermore, the processed sensor data displayed on the liquid crystal display and server or mobile phone. Data stored in the database that can reassess the patient's vital sign report. It is signalling system works through four processes: 1) retrieving data from sensors to raspberry pi, 2) storing vital sign data, 3) data visualization processes, 4) and making vital sign reports on servers or mobile phones. Figure 2 shows the design system of the vital sign. 


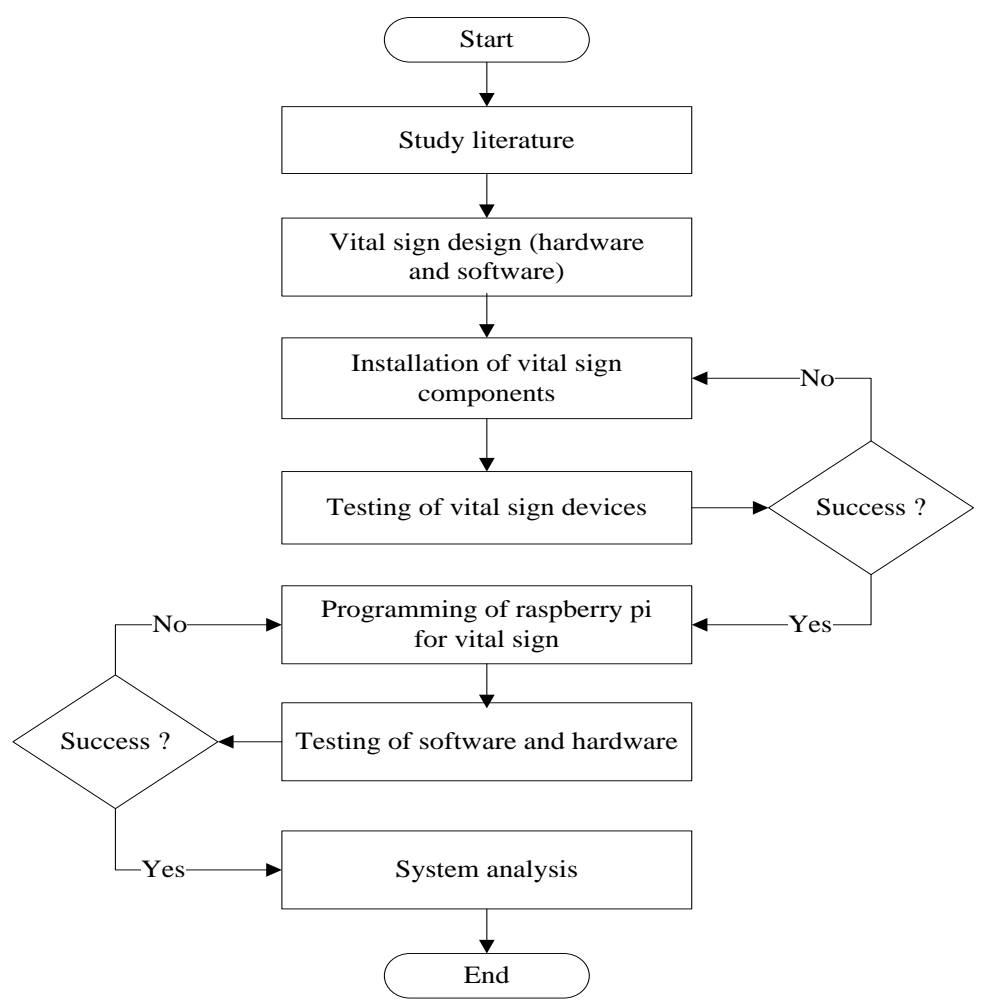

Fig. 1. Stage of research

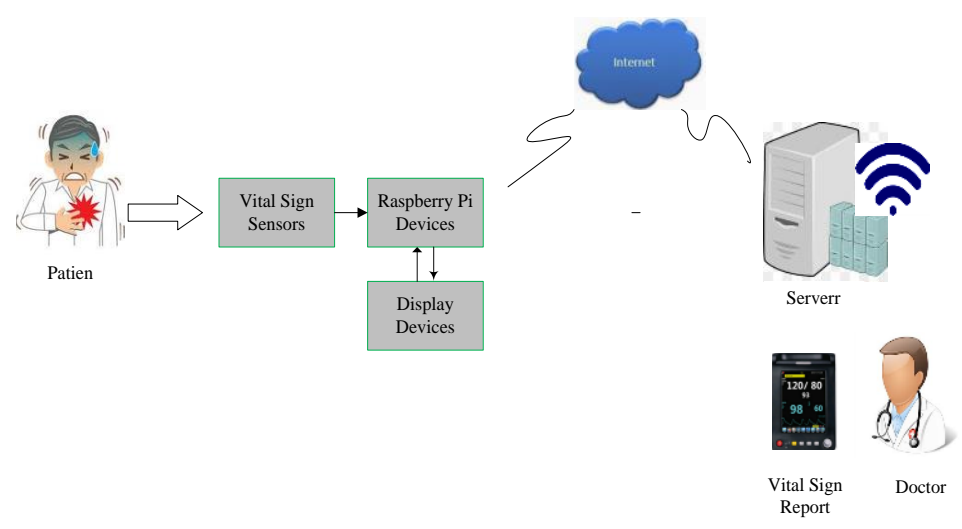

Fig. 2. Design system of the vital sign 


\subsection{Hardware design}

The design of vital sign hardware consists of power supplies, raspberry pi, Arduino, liquid crystal display (LCD), pulse sensor (HRM-2511E) and blood pressure sensor (MPX5050DP). The pulse sensor connected to Arduino and the temperature or blood pressure sensor connected to the raspberry pi. The heartbeat sensor data placed at one end of the finger and link to Arduino as a data processing module. The heartbeat produced based beats per minute (bpm). The data generated by the pulse sensor will be read and processed by raspberry pi and can be seen on the LCD. Figure 3 shows a diagram block of the vital sign.

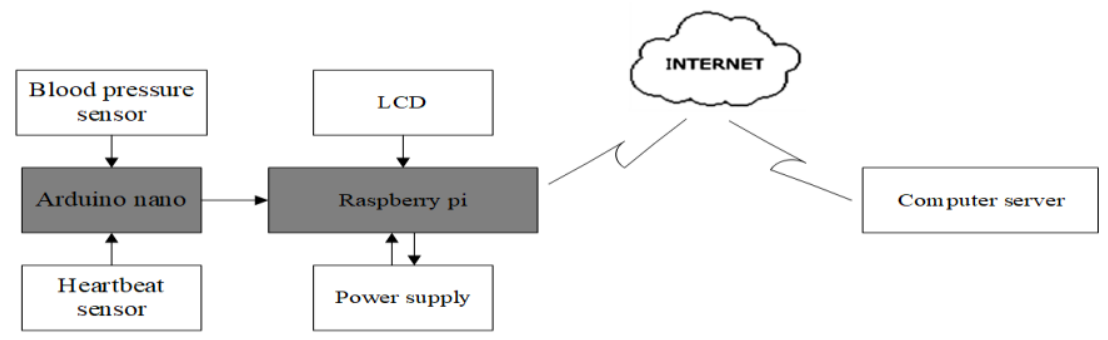

Fig. 3. Diagram block of the vital sign

\subsection{Software design}

This software design consists of readings and calculations to obtain the value of heartbeat and blood pressure. The next step is determining the condition of QS (heartbeat) worth true or false. The state of QS is true will continue in the heartbeat printing process. If the QS value becomes wrong, then return to the inspection process from the beginning. BPM value obtained from 1 minute divided by the average time between beats. The condition QS = true indicates that the BPM value has obtained. The Data results of the heartbeat or blood pressure will saved to the database and displayed on the LCD. In Arduino, there is an integrated development environment (IDE) program and uses the $\mathrm{C}$ programming language. The usefulness of the IDE application is to display sensor data and the text editor that has created. The text editor will be stored in the extension file .ino. Complete software design can see in Figure 4.

\subsection{Testing}

\subsubsection{Herabeat testing}

Tests were carried out by taking data from 10 patients with each sample 10 times. The purpose of this sampling is to obtain the average value of the calculation produced by the sensor. The results of the calculations will be compared with the readings from digital heartbeat device.

\subsubsection{Blood pressure testing}

Testing blood pressure sensors is a principle the same as the temperature and heart rate testing procedure. The blood pressure sensor will installed on the patient's arm. Each sample tested ten times. The data displayed is analog data from a blood pressure sensor that is converted to digital by the Arduino module. The calculation results obtained from blood pressure sensors compared with the values of blood pressure taken from digital 
sphygmomanometer. This value will use as a comparison with the blood pressure sensor designed. The display of the $\mathrm{mmHg}$ value on the LCD indicates that the sensor is working normally.

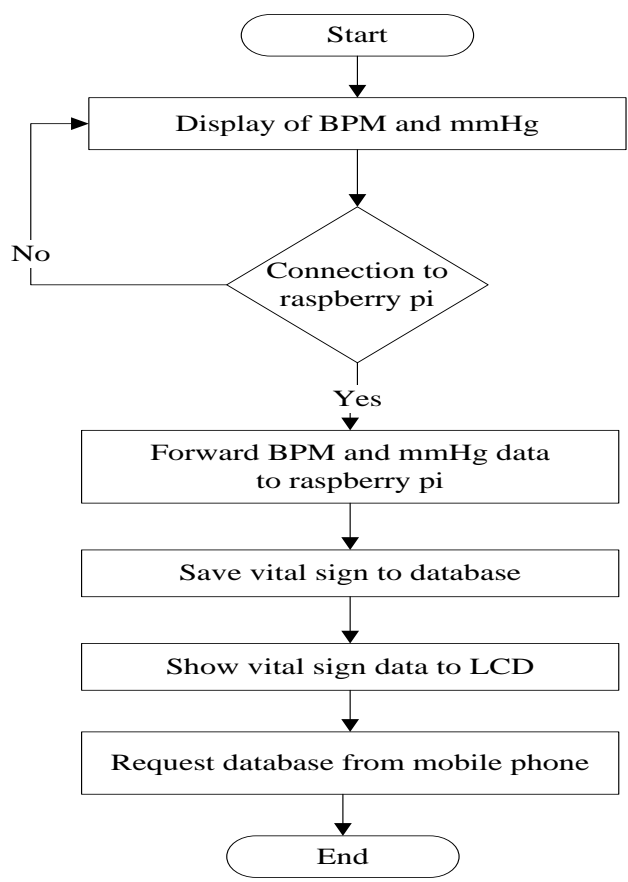

Fig. 4. Flowchart of software design

\subsubsection{Transmission Signal testing}

Testing of sending vital sign data done in two conditions. First is the state of open space without obstructions. Second is the condition of open space with an obstacle. In the overt area where the sensor is capable of sending data as far as 65 meters. In closed spaces where the sensor is capable of transmitting data as far as 12 meters. Tests for sending vital sign data can see in Figure 5 and 6.

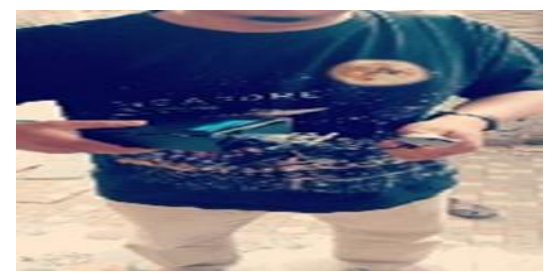

Fig. 5. Open space without an obstacle

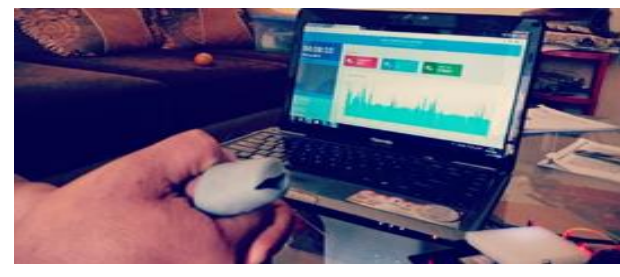

Fig. 6. Closed space with an obstacle 


\section{Results and Discussion}

\subsection{Retrieval of data}

\subsubsection{Retrieval of heartbeat data}

The purpose of measuring heart rate is to diagnose abnormalities in the heart that can affect heart attacks. The results of data collection were ten patients with a duration of 1 minute. The average heart rate produced is in the numbers 78-95 BPM. The values obtained are still categorized as normal because they are in the range of 60 - 100 BPM for adults 19 69 years. The design of the proposed heartbeat device works well, and the average error rate is $2.10 \%$.

Table 1. The data retrieval of heartbeat

\begin{tabular}{lcccc}
\hline No & Number of Patients & \multicolumn{2}{c}{ Blood Pressuret (mmHg) } & Error (\%) \\
& & HRM-2511E & Digital Heartbeat & \\
\hline 1. & 001 & 92 & 94 & 2.13 \\
2. & 002 & 86 & 89 & 3.37 \\
3. & 003 & 95 & 97 & 2.06 \\
4. & 004 & 90 & 93 & 3.23 \\
5. & 005 & 87 & 87 & 0 \\
6. & 006 & 78 & 82 & 4.88 \\
7. & 007 & 89 & 89 & 0 \\
8. & 008 & 89 & 91 & 2.19 \\
9. & 009 & 92 & 92 & 0 \\
10. & 010 & 91 & 94 & 3.19 \\
\hline \multicolumn{5}{r}{} \\
\hline
\end{tabular}

\subsubsection{Retrieval of blood pressure data}

The purpose of blood pressure measurements is to determine the condition of patient hypertension. The blood pressure threshold that categorized as the standard for adults is between $120 \mathrm{mmHg}$ of systole and $80 \mathrm{mmHg}$ of diastole. The results of data collection for ten patients got with an average value of 115 - $138 \mathrm{mmHg}$ (systole). The designed sensor performance works a normal with an average error rate of $2.31 \%$.

Table 2. The data retrieval of blood pressure

\begin{tabular}{lcccc}
\hline No & Number of Patients & \multicolumn{2}{c}{ Blood Pressure $(\mathrm{mmHg})$} & Error (\%) \\
& & MPX5050DP & Digital Device & \\
\hline 1. & 001 & 115 & 120 & 4.17 \\
2. & 002 & 120 & 125 & 4 \\
3. & 003 & 130 & 129 & 0.78 \\
4. & 004 & 138 & 136 & 1.47 \\
5. & 005 & 130 & 130 & 0 \\
6. & 006 & 125 & 125 & 0 \\
7. & 007 & 128 & 130 & 1.54 \\
8. & 008 & 128 & 130 & 1.54 \\
9. & 009 & 135 & 134 & 0.75 \\
10. & 010 & 120 & 120 & 0 \\
\hline
\end{tabular}




\subsubsection{Monitoring system of heartbeat and blood pressure}

On the monitoring system application menu vital sign is equipped with several facilities such as login and patient data entry. The process of vital sign data will be displayed after filling in data. The vital sign information presented in this application consists of values of body temperature, blood pressure, and heart rate. Explanation of patient data can see in Figure 7.

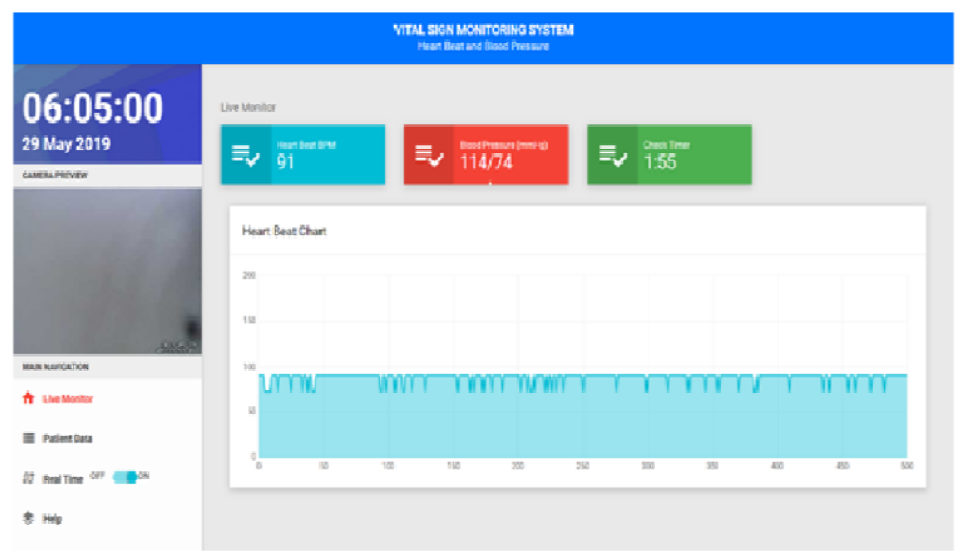

Fig. 7. Information of patient's data

Patient data that has processed stored on the raspberry pi device. The function of this device is to store data and send data to a notebook or mobile phone application. To bring up patient data stored in the database. Figure 8 shows the process of storing patient data.

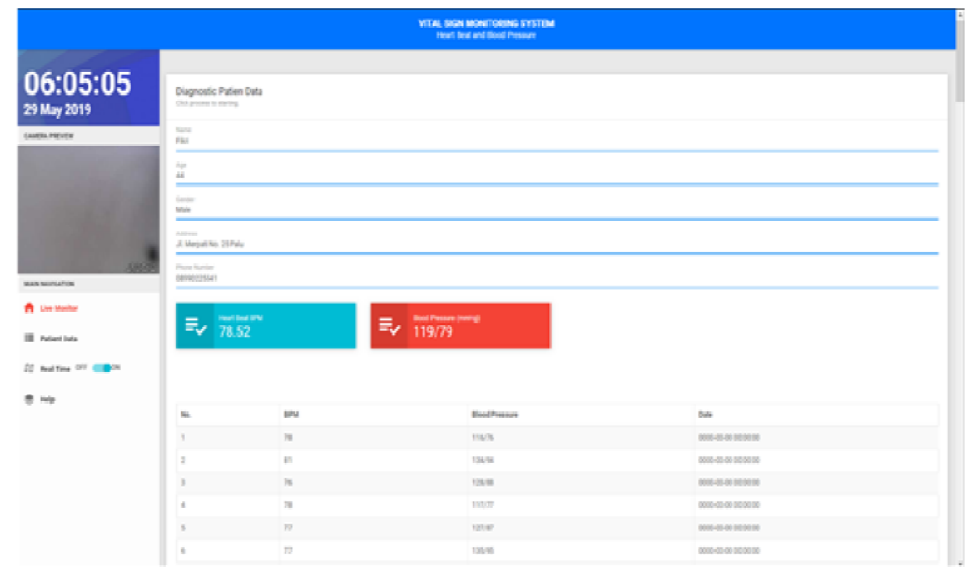

Fig. 8. Process data storage of patient's 


\section{Conclusion}

This research proposes a web-based vital sign monitoring system. Vital sign data consists of heart rate and blood pressure. It can display on a notebook or mobile phone in real time. The vital sign monitoring application designed to provide facilities to show patient databases based on the id-number. Based on the results of measurements and retrieval of vital sign data showed that the proposed tool worked well with an error rate for the blood pressure of $2.31 \%$ and heartbeat of $2.10 \%$.

\section{Acknowledgment}

Thank you to the Republic of Indonesia Research, Technology, and Higher Education (RISTEKDIKTI) have to provide financing support for the Applied Research Grant for 2019.

\section{References}

[1] C. C. Lin, M. J. Chiu, C. C. Hsiao, R. G. Lee, and Y. S. Tsai, "A wireless healthcare service system for elderly with Dementia, IEEE Trans. Inf. Technol,” Biomed, vol. 10, no. 4, pp. 696-704, 2006.

[2] E. Pearce, J. P. Smith, and P. A. Downie, A general textbook of nursing, Specialists and Other Experts. Faber, 1980.

[3] T. S. Sollu, Alamsyah, M. Bachtiar, and A. G. Sooai, "Patients' heart monitoring system based on wireless sensor network," in IOP Conference Series: Materials Science and Engineering, 2018, vol. 336, no. 1.

[4] P. E., "A $2.5 \mathrm{GHz}$ wireless ECG system for remotely monitoring heart pulses," in IEEE Antenna and Propagation Society (APS), 2012.

[5] T. S. Sollu, Alamsyah, M. Bachtiar, and B. Bontong, "Monitoring System Heartbeat and Body Temperature Using Raspberry Pi," E3S Web Conf., vol. 73, 2018.

[6] V. Wahane and P. V. Ingole, "An android based wireless ecg monitoring system for cardiac arrhythmia," in IEEE Healthcare Innovation Point-Of-Care Technologies Conference (HI-POCT), 2016.

[7] X. Sun and Y. Zhang, "Design and implementation of portable ecg and body temperature monitor," in International Symposium on Computer, Consumer and Control, 2014, pp. 910-913.

[8] Alamsyah, E. Setijadi, I. K. E. Purnama, and M. H. Purnomo, "Performance of the routing protocols AODV, DSDV and OLSR in health monitoring using NS3," in IEEE International Seminar on Intelligent Technology and Its Application (ISITIA), 2016.

[9] R. Tiwari, S. Shrivastava, and S. Das, "Performance evaluation of patient monitoring system with different routing protocols," Int. J. Comput. Appl., vol. 88, no. 14, pp. 2429,2014

[10] Alamsyah, E. Setijadi, I. K. E. Purnama, and M. H. Purnomo, "Performance analysis of AODV, AOMDV, and DSDV routing protocols in MANET based quality of service," in IEEE International Seminar on Application for Technology of Information and Communication. (iSemantic), 2018.

[11] W. Y. Chung, S. M. An, and S. C. Lee, "Real time multi-hop routing protocol for healthcare system based on WSN," in The 14th International Meeting on Chemical Sensors (IMCS), 2012, pp. 1253-1256.

[12] E. Chung, G. Chen, B. Alexander, and M. Cannesson, "Non-invasive continuous blood pressure monitoring: a review of current applications," Front. Med., vol. 7, pp. 91-101, 
2013.

[13] R. Chetney, "Home Care Technology and Telehealth," Wolters Kluwer Heal., vol. 21, no. 10, pp. 645-646, 2003. 